\title{
A Comprehensive overview of AR/VR by Writing in Air
}

\author{
Harneet Kaur"1 , Busireddy Guru Saran Reddy², Guna Chanukya Sai², Akula Srujan Raj² \\ ${ }^{*}$ Assistant Professor, Lovely Professional University, Jalandhar, Punjab, India \\ ${ }^{2}$ Research Scholar, Lovely Professional University, Jalandhar, Punjab, India
}

\begin{abstract}
Article Info

Volume 7, Issue 2

Page Number: 477-482

Publication Issue :

March-April-2021

\section{Article History}

Accepted : 20 April 2021

Published : 26 April 2021

The research is all about how often people could identify alphabets and numbers written in the open air. A leap motion captures motion trajectory information and plots it as a continuous stream of points. Lines would be combined and major slopes identified from the major points. Significant slopes are converted into directions by the use of geometry. Slope orientation is another name for this. Slope Alignment Sequence is a list of slope orientations that are used to individually depict Alphanumeric Letters. In this experiment, Dependency injection OpenCV is used to sketch on the camera with a virtual pen, i.e. any marker may be used to draw using the contour detection technique centered on the mask of the desired cultured reference marker. If you're a beginner who wants to enthuse your fellow members with a visual demonstration, the application user will be identified by the system until the produced slope direction sequence matches the system's comparison slope alignment sequence. In the algorithm's matching process, the sets and subsets process is used. The researchers suggested a new approach for classifying Alphabetic Chars written in the air in this article. The outcome of the experiment shows that the suggested approach is reliable.
\end{abstract}

Keywords : Alphabetic , OpenCV, Virtual Reality, Augmented Reality

\section{INTRODUCTION}

Air-writing is a form of input that helps a person to write or sketch in free space using their fingertips. Air-writing, like motion gestures, is recorded as a regular stream of information including a series of hand and finger motions. Air-writing differs from traditional writing in that the latter employs a paperpen-based writing system, while the former uses an abstract board in the air.
Unlike handwriting characters, those created in three-dimensional space lack the pen-up and quill detail that turns intra letters into sunstrokes. According to a review, air-drawn letter recognition is as effective as expression. The identification of airdrawn letters is not a simple sweep or rotating hand gesture but can represent different symbols. Writing in the air is sometimes used as a means of communication in this situation, particularly when interacting with those that are far away. 
"Many utilizing various types of sensors to reliably detect free-air motions in three-dimensional space. Yanmei and Chen et al" A.'s Actual Dynamic Handwriting Recognition Framework Using Sensor Module" is one example. Hand detection, data analysis, model training, and gesture classification are all part of this real-time Kinect-based dynamic HGR framework. As recognition algorithms, the Hidden Markov Model (HMM) and Support Vector Machine (SVM) are used. The identification rate for gestures 6 , $\mathrm{C}, \mathrm{O}$, and $\mathrm{V}$ was less than $50 \%$, bringing the overall average rate down to 82.86 percent of HMM. SVM, on the other hand, graded almost all of the movements as $100 \%$, but $60 \%$ on gesture $\mathrm{O}$, resulting in a 95.42 percent overall annual average". Tsuchida et alreport, "Written letter identification in the Air by Using Leap way of Controller," recognizes 46 Japanese Katakana symbols and 26 alphabets with an overall recognition rate of 86.7 percent, with 84.54 percent precision for recognizing the English alphabet. Besides, in 2015, Jayesh Kumar Sharma et al. published "Numeral Gesture Recognition with Leap Motion Sensor," and was using the Mathematical Face Recognition device and the Leap Cellular Modem to track free air digit gestures from 0-9. Position spotting, feature recognition, gesture tracking, preparation, and classification were the three phases of the method.

The algorithm's drawback, on the other hand, is that it fails to recognize expressions with an open curve. As a result, the system was only able to reach a classification rate of 70.2 percent. The main issue in previous studies was lettered with curves, especially open curves. Other recent experiments have issues with the letter's size and form. The system's consistency rating continues to drop as a result of misclassified letters.

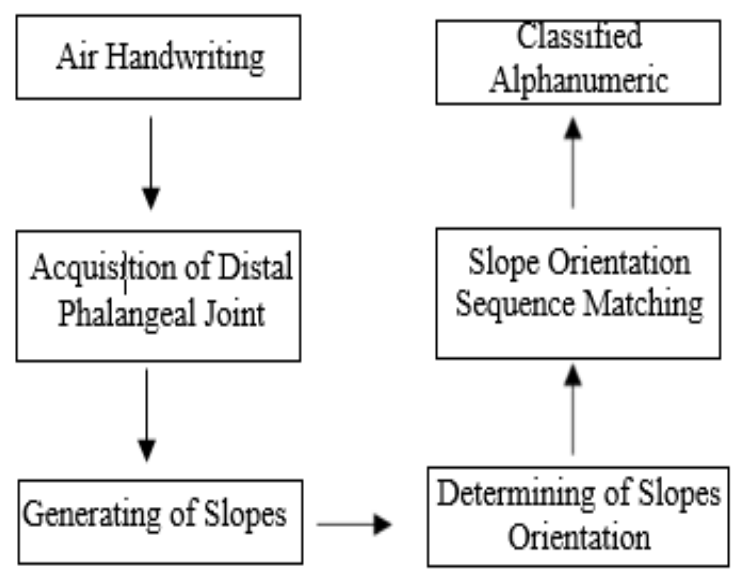

Figure 1: Flow Chart

The researchers suggest a recognition scheme based on the Slope Orientation Sequence Matching Algorithm to address an issue that has been identified in previous studies. The entire function of the machine is shown in the system block diagram. It contains portable air as the system input, acquisition of human hand distal phalange joint coordinates, generation of slopes and angles created, slope orientation determination, slope orientation matching, and graded numeric values as the system output. One of the most cutting-edge technologies in the area of personal communication is air-writing technical environmental. As a result, the research would be very useful in the scholarly and medical fields. The suggested algorithm also introduces a novel idea of complex sign language recognition, which can be used in future research.

\section{LITERATURE REVIEW}

Recognizing multi thumb is a difficult and open topic in computer display due to the different issues addressed in the previous segment. The list consists of previous research that is relevant to this project: Plane recognition fingertip detection and monitoring, as well as existing hand tools related to air-drawing information acknowledgment Fingertip Detection method Based In the fields of human-computer connectivity and virtual reality, fingertip detection, 
and tracking use color images cameras has been a hot topic (AR). As a preliminary phase for fingertip detection, a model-based technique four for 3Dtechnic hand tracking (de La Gorce et al. Tang et al) used however, these approaches are computationally expensive and necessitate a large volume of training data. As a consequence, our real-time programming is incompatible with them. After the hand is subdivided using color, working depth, or movement cues, the tips are viewed from the derived binary hand shield in the version scheme. Liang and his colleagues [6] used a range metric from either the back of the hand to both the contour's furthermost points to find candidate fingertip marks. Krejov and Bowden [7] generalized the distance principle by using a geodesic distance for the normal structure of the side. In hand setups where previous techniques failed, this increased the localization of fingertips. Since fingertips have a higher curvature than other parts of the hand, the authors of (Lee and Hollered, 2007) used the contour curvature as a signal to spot fingertips.

Inside the device's interaction field, the Motion Sensor has a skeletal tracking function that depends on individual fingertips. As a result, the finger's skeletal joint coordinates can be obtained. As a result, the distal phalange joint coordinates of the human hand are acquired using the Leap Motion Controller sensor. The device can map points and gain coordinates when the user enters an alphanumeric character in free space. "There are many uses for automatic object recognition, including machine learning and social contact. Objects can range from a text to a person being that needs to be monitored. The monitoring algorithm has been implemented in a variety of ways in the literature. Another number of experts used it to decode Sign Languages another for hand gesture recognition, and yet another for text translation and detection, tracing complete body movement of items for virtual reality, and yet another for fingerprint tracking dependent character recognition".

Neumann and colleagues devised a system for text identification and localization in real-world images. They used a hypothesis system in their paper that can process several text lines. They also train the algorithm with synthetic scripts, and they take advantage of the Maximally Legitimate Power Regions (MSERs), which provide reliability to geometric but important for quality [8]. Wang et al. have explored a color-based body movement method for both interior and exterior settings in [10]. To detect the item, they used a recording device and a colored shirt in their proposed process. The results of their proposed approach suggest that it can be used in augmented reality implementations.

Objects can range from a text to a person being that needs to be monitored. The monitoring algorithm has been implemented in a variety of ways in the literature. Another number of experts used it to decode Sign Languages, another for hand gesture recognition, and yet another for text translation and detection, tracing complete body movement of items for virtual reality [10], and yet another for fingerprint tracking dependent character recognition. The writers of talk about a visual guy that can recognize Japanese katakana characters in the breeze. They used a TV camera and a Light Emission Diode (LED) pen to monitor the hand gesture. They translate pen action into path codes. To remove the impact of writing speed, the codes are normalized to 100 data pieces, with 46 Japanese characters specified. They reach 92.9 percent character recognition accuracy with a single camera and 9 percent gesture path accuracy with multiple cameras.

As previously said, our suggested approach works on the color portion of the frame. It also monitors the whole area for it. Two characters could be 
distinguished by using tape only on the frontal portion of the right thumb, and after writing every letter, simply rotate the finger (where no tape is present) and write the next letter. When the proposed procedure fails to locate a colored item, it is concluded that one of the characters has been achieved. Another option is to add a delay until each character is written. The waiting time will be $\mathrm{m}$ seconds, resulting in duplicate frames of the same features. As previously said, we quantify the difference between two images using the previous frame as a reference image. The smaller the discrepancy value, which in the case of reproduced frames (coordinates of $\mathrm{x}, \mathrm{y}$ ), is normally equivalent to zero, the more accurate one letter is. Both statements are undeniably right.

\section{METHODOLOGY}

The Leap Remote Control has a skeletal tracking system that focuses on the individual fingers throughout the device's contact region. As a result, the finger's skeletal joint coordinates can be obtained. As a result, the distal micro lading socket coordinates of the human hand are acquired using the Leap Motion Controller sensor.

Every stroke is made up of a series of points from which the machine software selects the main points that will reflect the stroke. The sudden shift in slope $\mathrm{n}$ is used to determine major points. Three trials were performed to assess the acceptable tolerance for slope $\mathrm{n}$ in obtaining the major points to be considered in the study. The smallest agreed value of shift between slopes $\mathrm{n}$ is called tolerance.

\section{IMPLEMENTATION}

The slope formula is then applied to the two consecutive main points, which are made up of $\mathrm{x}$ and $\mathrm{y}$ coordinates. The value of change in $\mathrm{x}$-coordinates and change in $\mathrm{y}$-coordinates determines the slope of a line. The horizontal length of the line formed by two consecutive major points is defined by the change in $\mathrm{x}$-coordinates, while the vertical length is determined by the change in $y$-coordinates.

The slope orientation is determined by the acquired slope, which is classified into eight categories: Upward (U) Right (R) Downward (D) Left (L) RightUp (RU), Absolutely correct (RD), Decided to leave (LD), and National democratic (LU) (LU). Straight lines are difficult to input while using a leap motion sensor. As a result, the researchers looked at the average variety of angles used by five (5) users when drawing straight lines - up, right, down, and left - in five (5) trials each.

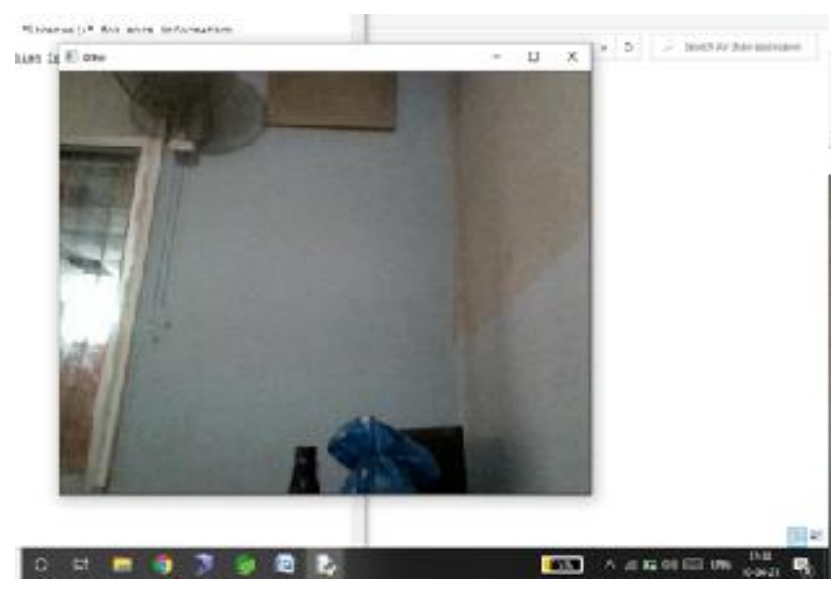

Figure 2: Screen1

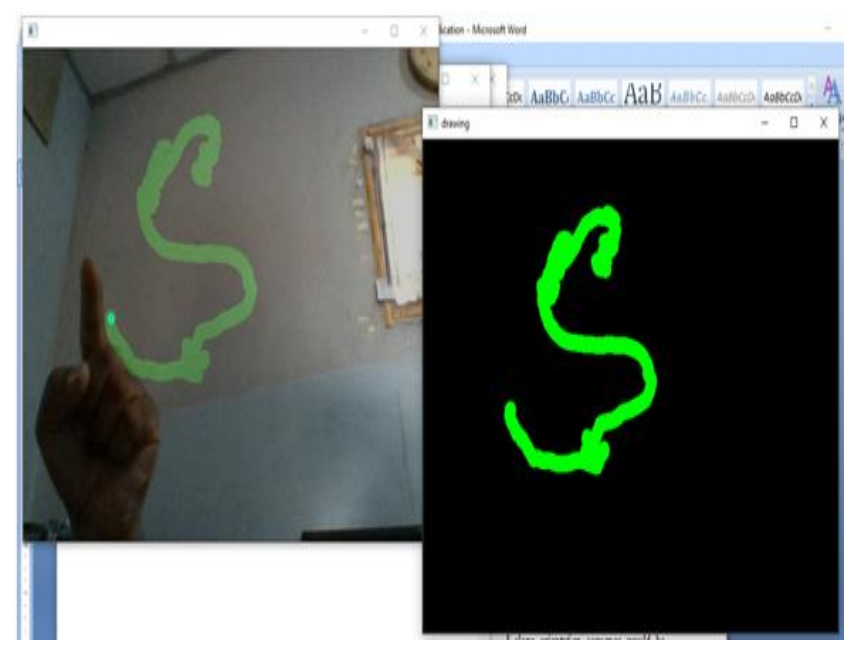

Figure 3: Screen 2 
You'll see a text that says "Draw: $s$ " in the first detection panel. The number "s" is created at random, and you must draw it on the canvas. Since the 'object' is a green bottle-cap, a text will appear at the top of the webcam that says, "Object Found." Another text would appear in the lower region of the detection screen, indicating the object's area. The size of the pen will be proportional to the size of the area. To change the pen height, move the environmentally friendly object nearer to the camera (to increase) or further away from the webcam (to decrease).

\section{RESULTS AND DISCUSSION}

The researchers performed 25 trials per character to determine the slope alignment pattern to be used in describing each rot, and a comparison of the most common sequences for each letter Straight line characters have several different slope orientation sequences; each character may have up to four different slope orientation sequences. The user's writing style is to blame for this. "The majority of users tend to draw characters in a range of sizes and strokes. Because of the sensitivity of the Leap Motion Controller sensor, straight lines are often difficult to achieve. It is critical to include several sets of slope orientation sequences for some alphanumeric characters to increase system recognition rates. Since 0 and $O$ have had the same slope sequence, the user should draw numbers with two fingers and letters with one finger to prevent misclassification". Letters $\mathrm{P}$ and $\mathrm{D}$, as well as $\mathrm{U}$ and $\mathrm{V}$, have almost equivalent slope series, participating in misidentification.

\section{CONCLUSION}

The slope direction series obtained from the airdrawn alphanumeric characters are distinctly based on the gathered data. However, certain alphanumeric character slope orientation sequences are almost identical. Letters $\mathrm{U}$ and $\mathrm{V}$, as well as letters $\mathrm{D}$ and $\mathrm{P}$, have similar slope orientation sequences in this sense. Since these characters have the same characteristics, the produced slope orientation sequence would be identical. Characters with a large number of strokes, but on the other hand, formed distinct lines of comparison slope inclination series, as seen on characters 5, B, E, F, K, and M. The key criterion for acquiring slopes is the gap threshold. The number of slopes is inversely proportional to the distance threshold. As a consequence, the lower the cultural boundaries, the more slopes are acquired; thus, the shorter the slope orientation series. The Slope Orientation Sequence Matching Algorithm can also be used to recognize the writing of alphanumeric characters in free air, according to the data. The algorithm, on the other hand, is based on the sequence of slopes created, making the method timedependent. The result demonstrates the character's inconsistencies in terms of the number of slopes as well as the slope series composition. As a result, certain characters with several meanings, such as $\mathrm{E}$ and F, are misclassified by the system. The slopes shaped when writing the letters $U$ and $V$ are mirrored by the latter due to the same motion. As a consequence, the method incorrectly classifies $\mathrm{U}$ as $\mathrm{V}$. The 'webcam paint' will be run again and the trial number will be increased if you click the Continue button. The resulting panel displays the assigned number (which is created at random), as well as the prediction of your drawing and the points you earned during the trials.

\section{REFERENCES}

[1]. Alper Yilmaz, Omar Javed, Mubarak Shah, "Object Tracking: A Survey", ACM Computer Survey. Vol. 38, Issue. 4, Article 13, Pp. 1-45, 2006 Taylor \& Francis Journal of Information

Display Volume 14, Issue 4, 2013 DOI:10.1080/15980316.2013.860928 Ref: ] 
http://learnrnd.com/news.php?id=Swarm_Clot hing:Dress_Changes_Automatically_

[2]. Yuan-Hsiang Chang, Chen-Ming Chang, "Automatic Hand-Pose Trajectory Tracking System Using Video Sequences", INTECH, pp. 132- 152, Croatia, 2010

[3]. Erik B. Sudderth, Michael I. Mandel, William T. Freeman, Alan S. Willsky, "Visual Hand Tracking Using Nonparametric Belief Propagation", Mit Laboratory For Information \& Decision Systems Technical Report P-2603, Presented at IEEE CVPR Workshop On Generative Model Based Vision, Pp. 1-9, 2004

[4]. Robert Y. Wang, Jovan Popovi'c, "Real-Time Hand-Tracking with a Color Glove”, 2008

[5]. T. A. C. Bragatto, G. I. S. Ruas, M. V. Lamar, "Real-time Video Based Finger Spelling Recognition System Using Low Computational Complexity Artificial Neural Networks", IEEE ITS, pp. 393-397, 2006

[6]. Yusuke Araga, Makoto Shirabayashi, Keishi Kaida, Hiroomi Hikawa, "Real Time Gesture Recognition System Using Posture Classifier and Jordan Recurrent Neural Network", IEEE World Congress on Computational Intelligence, Brisbane, Australia, 2012

[7]. Ruiduo Yang, Sudeep Sarkar, "Coupled grouping and matching for sign and gesture recognition", Computer Vision and Image Understanding, Elsevier, 2008

[8]. http://learnrnd.com/news.php?id=How_Printin g_Batteries_Made,_Its_Structure_Usage_and_F utur e_Applications

[9]. Jari Hannuksela, Sami Huttunen, Pekka Sangi, Janne Heikkila, "Motion-Based Finger Tracking For User Interaction with Mobile Devices" In Proc. 4th Eur. Conf. Vis. Media Production, pp. 1-6, London, U.K., 2007.

[10]. H.M. Cooper, "Sign Language Recognition: Generalising to More Complex Corpora", $\mathrm{PhD}$ Thesis, Centre for Vision, Speech and Signal
Processing Faculty of Engineering and Physical Sciences, University of Surrey, UK, 2012

[11]. Kim, Sung Deuk, Jaeyoun Yi, Hyun Mun Kim, and Jong Beom Ra. "A deblocking filter with two separate modes in block-based video coding." Circuits and Systems for Video Technology, IEEE Transactions on 9, no. 1 (1999): 156-160

[12]. Buckingham, Michael J., Broderick V. Berknout, and Stewart AL Glegg. "Imaging the ocean with ambient noise." Nature 356, no. 6367 (1992): 327-329.

[13]. Vaseghi, Saeed V. Advanced digital signal processing and noise reduction. Wiley, 2008.

[14]. Plataniotis, Konstantinos N., and Anastasios N. Venetsanopoulos. Color image processing and applications. Springer, 2000.

\section{Cite this article as :}

Harneet Kaur, Busireddy Guru Saran Reddy, Guna Chanukya Sai, Akula Srujan Raj, "A Comprehensive overview of AR/VR by Writing in Air", International Journal of Scientific Research in Computer Science, Engineering and Information Technology (IJSRCSEIT), ISSN : 2456-3307, Volume 7 Issue 2, pp. 477-482, March-April 2021. Available at doi : https://doi.org/10.32628/CSEIT217294 Journal URL : https://ijsrcseit.com/CSEIT217294 